\title{
Rank, Remit, and Resources
}

As indicated in the title, there are three Rs to be covered in this editorial, starting with Rank. Our current 2019 Journal Impact Factor has increased to 3.884 (Journal Citation Reports, 2020 Clarivate Analytics), placing International Journal of Sport Nutrition and Exercise Metabolism (IJSNEM) among the top few journals in our field. This is a testament both to the high quality of work from authors publishing with us and to the efforts of our editorial team under the leadership of Editor-in-Chief Emeritus, Professor Ronald Maughan. My hope is that authors will continue to submit valuable research to this journal, which we can then publicize to ensure that the work gains attention and achieves impact. To fulfill those objectives, articles accepted at IJSNEM are published promptly-typically now appearing in the next but one issue. We also select one lead paper and one free open access paper from each new issue (along with a "classic" paper from the IJSNEM archives), which are all made publicly available and featured via our social media networks and growing collection of infographics (courtesy of www.ylmsport science.com).

Second, in terms of Remit, IJSNEM has always encompassed papers from a variety of disciplines but, consistent with our stated mission and scope, the primary aim of this journal is to provide an outlet for articles relating to the biochemical, physiological, and molecular aspects of sports nutrition and exercise metabolism. Nonetheless, we receive many submissions describing crosssectional surveys of intangible constructs that can be difficult to objectively quantify (e.g., knowledge, attitudes, or beliefs about nutrition); publication of such articles should be the exception rather than the rule at IJSNEM, and to be an exception, these studies must avoid the limitations that seem particularly common and problematic for research of that nature. Specifically, while almost all human research understandably involves a self-selected (i.e., nonrandom) sample of willing volunteers, this arguably poses the greatest threat to generalizability if the primary outcome is predictive of participation (e.g., Are volunteers for nutrition knowledge surveys especially interested/knowledgeable about nutrition?). One approach to this problem is to use a large and representative sample, but this typically involves online surveys or self-reported diet records, which do not generally provide the desired degree of control over the data collection (i.e., Did volunteers respond accurately, honestly, and independently?). Even assuming that diet is recorded effectively, many papers then express nutritional intake in terms of dietary adequacy or nutrient availability/status, yet metabolic requirements are rarely measured and so net balances are too often unreliable estimates. This journal therefore continues to encourage submissions that address important questions using cross-sectional surveys but, to be suitable for publication in IJSNEM, we must be confident that the observed data provide a true reflection of the study sample and that inferences can be drawn beyond that sample.

Finally, under the heading of Resources, I am pleased to share two new tools provided in the "Author Toolbox" section of the journal website under "Author Guidelines." To assist authors in preparing their manuscripts, we now provide a document template consistent with the formatting requirements of this journal (with links in each section to our PRESENT 2020 submission checklist; Betts et al., 2020). Moreover, the September issue of IJSNEM includes a paper introducing our newly developed Time Series Response Analyser (TSRA), which was created by Benjamin Narang to automate the process of reducing time series data to relevant summary statistics (e.g., incremental area under the curve; Narang et al., 2020). Anyone who has manually calculated such measures will be all too aware that the process can be timeconsuming and prone to errors in both computation and choice of methodology. The TSRA efficiently, accurately, and transparently implements standardized procedures for deriving common summary statistics, while also offering a range of alternative approaches to data analysis and visualization that may reveal important patterns in temporal data (e.g., peak/nadir values, time to peak, variability, response exceeding clinical thresholds). I have no doubt that this resource will be useful for contributors to IJSNEM and will reassure readers that the summary statistics reported in this journal are correctly calculated. We are happy for the TSRA to be circulated freely and included with the submission guidelines of any other journals.

James A. Betts, University of Bath

\section{References}

Betts, J.A., Gonzalez, J.T., Burke, L.M., Close, G.L., Garthe, I., James, L.J., . . Atkinson, G. (2020). PRESENT 2020: Text expanding on the checklist for proper reporting of evidence in sport and exercise nutrition trials. International Journal of Sport Nutrition and Exercise Metabolism, 30(1), 2-13. doi:10.1123/ijsnem.2019-0326

Narang, B.J., Atkinson, G., Gonzalez, J.T., \& Betts, J.A. (2020). A tool to explore discrete-time data: The Time Series Response Analyser. International Journal of Sport Nutrition and Exercise Metabolism, 30(5), 374-381. doi:10.1123/ijsnem.2020-0150 